\title{
Microwave background temperature at a redshift of 6.34 from $\mathrm{H}_{2} \mathrm{O}$ absorption
}

https://doi.org/10.1038/s41586-021-04294-5

Received: 12 February 2021

Accepted: 30 November 2021

Published online: 2 February 2022

Open access

Check for updates

\author{
Dominik A. Riechers ${ }^{1 凶}$, Axel Weiss ${ }^{2}$, Fabian Walter ${ }^{3,4}$, Christopher L. Carilli $^{4}$, Pierre Cox ${ }^{5}$, \\ Roberto Decarli ${ }^{6}$ \& Roberto Neri ${ }^{7}$
}

Distortions of the observed cosmic microwave background provide a direct measurement of the microwave background temperature at redshifts from 0 to 1 (refs. ${ }^{1,2}$ ). Some additional background temperature estimates exist at redshifts from 1.8 to 3.3 based on molecular and atomic line-excitation temperatures in quasar absorption-line systems, but are model dependent ${ }^{3}$. No deviations from the expected $(1+z)$ scaling behaviour of the microwave background temperature have been seen ${ }^{4}$, but the measurements have not extended deeply into the matter-dominated era of the Universe at redshifts $z>3.3$. Here we report observations of submillimetre line absorption from the water molecule against the cosmic microwave background at $z=6.34$ in a massive starburst galaxy, corresponding to a lookback time of 12.8 billion years (ref. ${ }^{5}$ ). Radiative pumping of the upper level of the ground-state ortho- $\mathrm{H}_{2} \mathrm{O}\left(1_{10}-1_{01}\right)$ line due to starburst activity in the dusty galaxy HFLS3 results in a cooling to below the redshifted microwave background temperature, after the transition is initially excited by the microwave background. This implies a microwave background temperature of $16.4-30.2 \mathrm{~K}$ (1 $1 \sigma$ range $)$ at $z=6.34$, which is consistent with a background temperature increase with redshift as expected from the standard $\Lambda$ CDM cosmology ${ }^{4}$.
We used the Northern Extended Millimeter Array (NOEMA) to obtain a sensitive scan across the $3-\mathrm{mm}$ atmospheric window towards the $z=6.34$ massive dusty starburst galaxy HFLS3 (also known as 1HERMES S350 J170647.8+584623; see Methods) $)^{5}$. These observations reveal a broad range of emission features dominated by the $\mathrm{CO}, \mathrm{H}_{2} \mathrm{O}$ and $\mathrm{H}_{2} \mathrm{O}^{+}$ molecules and atomic carbon, on top of thermal dust continuum emission that is rising with frequency consistent with a dust temperature of $T_{\text {dust }}=63.3_{-5.8}^{+5.4} \mathrm{~K}$ (refs. ${ }^{5,6}$ ) (Fig. 1 ). The spectrum also shows a deep absorption feature due to the ortho- $\mathrm{H}_{2} \mathrm{O}\left(1_{10}-1_{01}\right)$ ground-state transition at rest-frame $538 \mu \mathrm{m}$ (observed at $3.95 \mathrm{~mm}$, or $75.9 \mathrm{GHz}$ ). This absorption is about two times stronger than the continuum emission from the starburst at the same frequency (Fig. 2). For this effect to occur, a substantial population of the ortho- $\mathrm{H}_{2} \mathrm{O} 1_{10}$ level (which lies 26.7 Kabove the $1_{01}$ ground state) has to be excited by cosmic microwave background (CMB) photons as a basis for pumping of this level by the starburst infrared radiation field (see Extended Data Fig. 1). The effect becomes observable towards HFLS3 because of the warm CMB at this redshift, which is predicted to be $T_{\mathrm{CMB}}=20.0 \mathrm{~K}$ at $z=6.34$ based on the standard $\Lambda$ CDM cosmology (where $T_{\mathrm{CMB}}(z)=T_{\mathrm{CMB}}(z=0)^{*}(1+z)^{(1-\beta)}, T_{\mathrm{CMB}}$ $(z=0)=2.72548 \pm 0.00057 \mathrm{~K}$ ( ref. $\left.^{7}\right)$ and the power-law index $\left.\beta=0\right)$. The absorption of photons from the CMB radiation field appreciably populates the $\mathrm{H}_{2} \mathrm{O}_{10}$ level. The intense infrared radiation field from the starburst then preferentially de-populates the $1_{10}$ level through radiative pumping, resulting in a deficit in the $1_{10}$ level compared with $1_{01}$ relative to a thermal distribution. In combination, these two processes result in an excitation temperature $T_{\mathrm{ex}}$ of the $\mathrm{H}_{2} \mathrm{O}\left(1_{10}-1_{01}\right)$ line that is lower than $T_{\mathrm{CMB}}$, such that the line becomes observable in absorption against the $\mathrm{CMB}$. As the effect depends on the strength of the $\mathrm{CMB}$ radiation field, it can be used to measure $T_{\mathrm{CMB}}$ for galaxies that have well-measured dust spectral energy distributions and dust continuum sizes, as is the case for HFLS3.

To understand the effect, we have calculated a series of spherically symmetric RADEX ${ }^{8}$ models over a wide range of $\mathrm{H}_{2} \mathrm{O}$ column densities, assuming purely radiative excitation (Figs. 2 and 3; see Methods for additional details). Exposing a cold, $\mathrm{H}_{2} \mathrm{O}$-bearing region associated with HFLS3 to the black-body CMB radiation field at $T_{\mathrm{CMB}}(z=6.34)$, the models suggest that $77.2 \%$ of the molecules will be in the $1_{01}$ ground state and $20.3 \%$ will be in the upper $1_{10}$ state, and all $\mathrm{H}_{2} \mathrm{O}$ transitions have an excitation temperature $T_{\mathrm{ex}}$ equal to $T_{\mathrm{CMB}}$. As a result of this zero temperature contrast, no $\mathrm{H}_{2} \mathrm{O}$ emission or absorption would be observable, despite the fact that the $\mathrm{H}_{2} \mathrm{O}$ rotational ladder is excited by the $\mathrm{CMB}$ radiation. However, this picture changes when the same region is also exposed to the infrared radiation field emitted by the starburst nucleus of HFLS3, as the latter does not follow a single-black-body radiation pattern. Indeed, the infrared spectral energy distribution of HFLS3 reaches its peak intensity at $73.3_{-1.3}^{+1.6} \mu \mathrm{m}$ and can be approximated by a grey body with a Rayleigh-Jeans slope of $\beta_{\mathrm{IR}}=1.94_{-0.09}^{+0.07}$. This is due to the presence of dust at multiple temperatures and an increasing dust optical depth towards shorter wavelengths $s^{5,6}$. In this case, the level populations of the $1_{01}$ and $1_{10}$ states will deviate from the

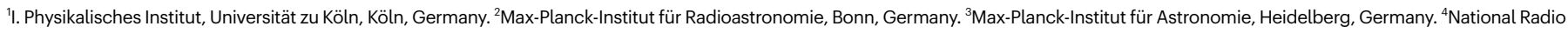
Astronomy Observatory, Pete V. Domenici Array Science Center, Socorro, NM, USA. ${ }^{5}$ Sorbonne Université, UPMC Université Paris 6 and CNRS, UMR 7095, Institut d'Astrophysique de Paris, Paris, France. ${ }^{6}$ INAF - Osservatorio di Astrofisica e Scienza dello Spazio, Bologna, Italy. Institut de Radioastronomie Millimetrique, Saint Martin d'Héres, France. ${ }^{\circledR}$ e-mail: riechers@ph1.uni-koeln.de 


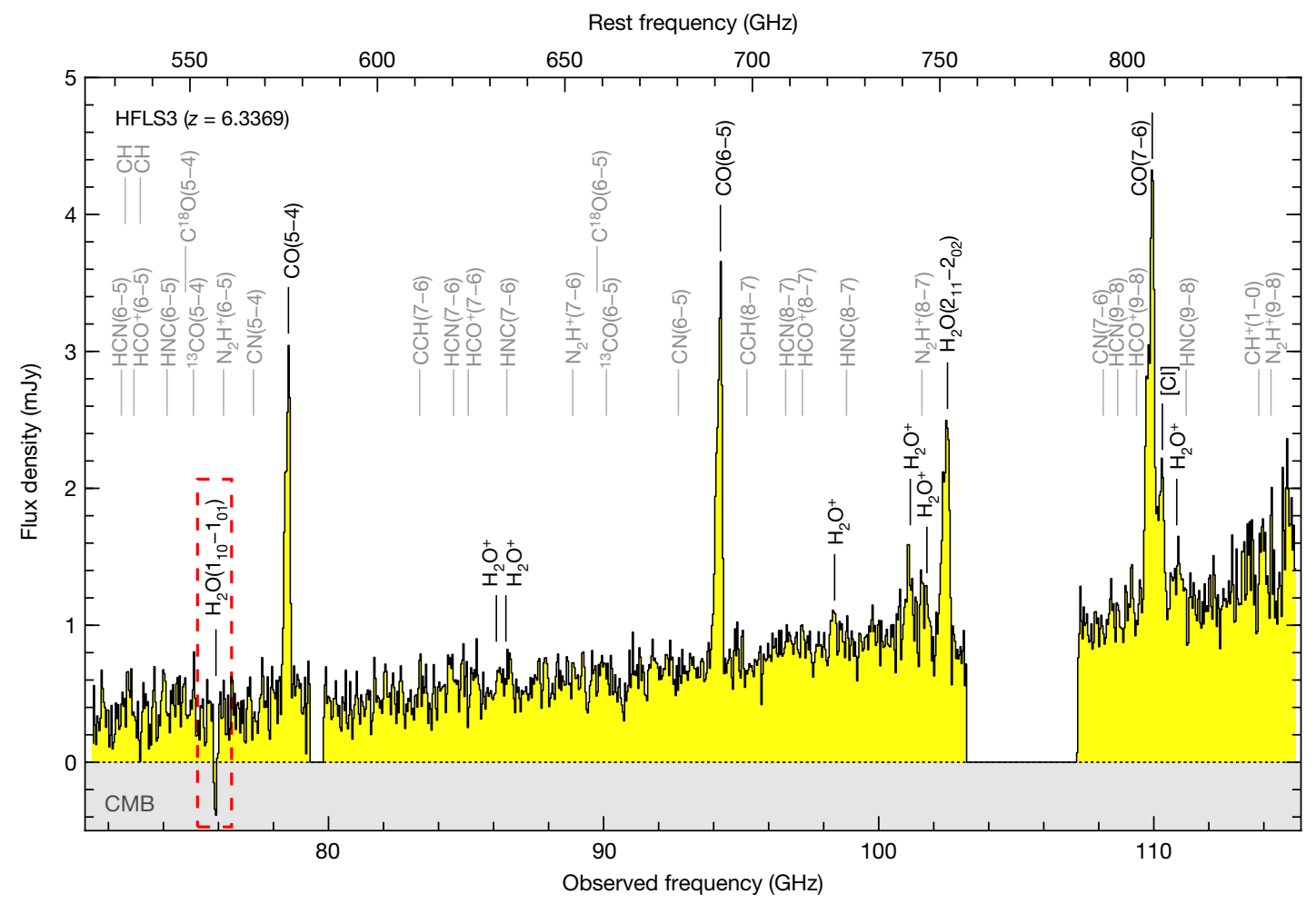

Fig. 1 | Broad-band, 3-mm spectroscopy of the starburst galaxy HFLS3 at a redshift of 6.34 with NOEMA. Black/yellow histogram, NOEMA spectroscopy data, binned to $40-\mathrm{MHz}\left(158 \mathrm{~km} \mathrm{~s}^{-1}\right.$ at $\left.75.9 \mathrm{GHz}\right)$ spectral resolution. Expected frequencies of molecular and atomic lines at the redshift of HFLS3 are indicated, with the dominant species labelled in black. The dashed red box indicates the frequency range of the ortho- $\mathrm{H}_{2} \mathrm{O}\left(1_{10}-1_{01}\right)$ line, which is detected in absorption against the CMB. single-temperature thermal equilibrium population and change to $68.0 \%$ and $14.6 \%$, respectively, for the solution shown in Fig. 2, resulting in an excitation temperature $T_{\text {ex }}$ of only $17.4 \mathrm{~K}$ for this transition. Owing to the $\Delta J=1$ selection rule for photon emission/absorption, only three ortho- $\mathrm{H}_{2} \mathrm{O}$ transitions contribute to the modification of populations in the $1_{01}$ and $1_{10}$ levels, namely, the $538-\mu \mathrm{m} 1_{10}-1_{01}$ and $180-\mu \mathrm{m} 2_{12}-1_{01}$ transitions affecting the former, and the $108-\mu \mathrm{m} 2_{21}-1_{10}$ transition affecting the latter (see Fig. 2 ; the $2_{21}-1_{01}$ transition is forbidden).
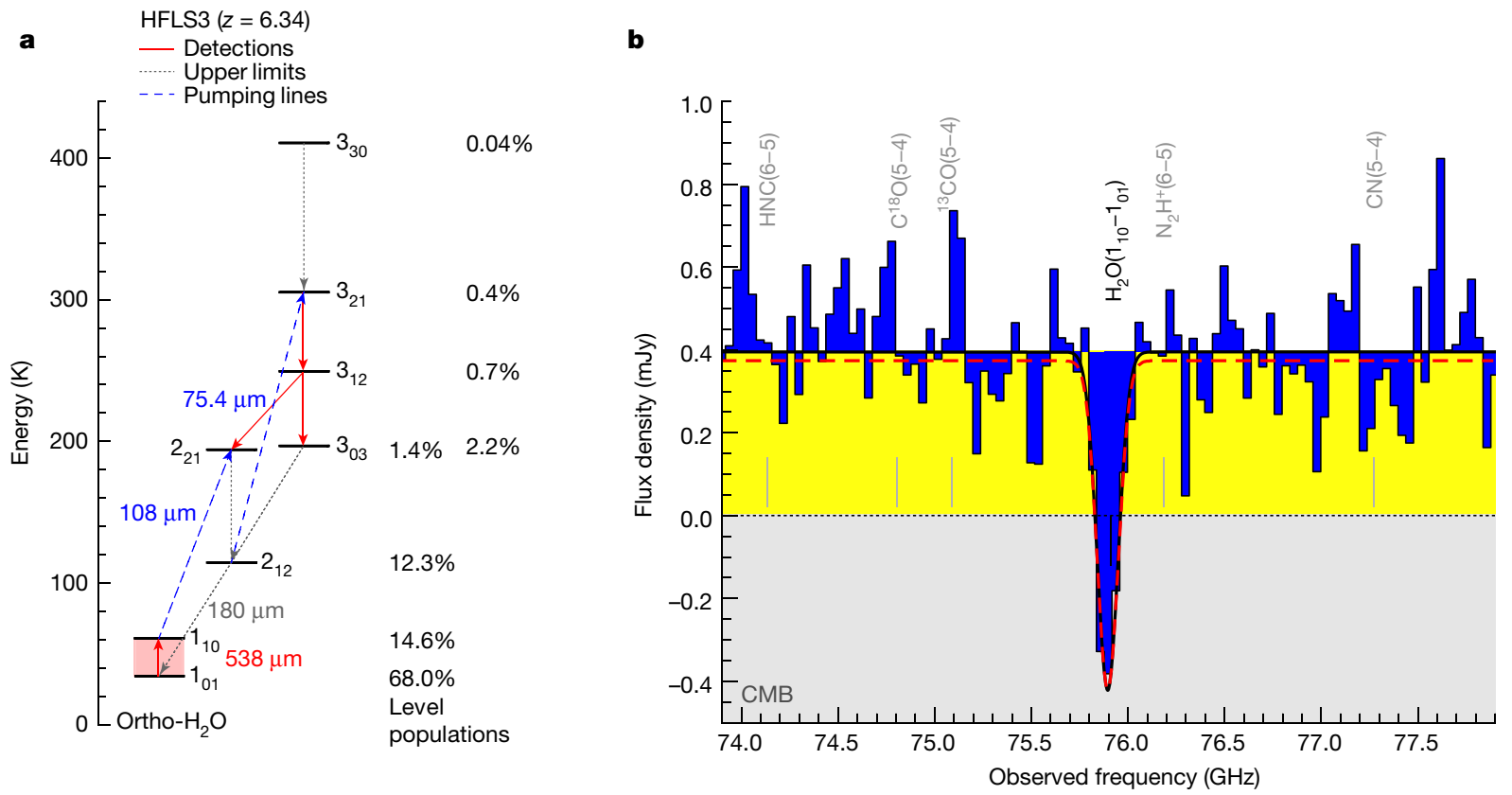

Fig. $2 \mid \mathrm{H}_{2} \mathrm{O}$ line and continuum properties and modelling of HFLS3. Ortho- $\mathrm{H}_{2} \mathrm{O}$ energy-level structure (a, red solid arrows are detected transitions, grey dotted lines are upper limits and blue dashed arrows are pumping transitions, with observed and model-predicted absorption/emission lines indicated as upward/downward arrows, respectively; percentages are the level populations in the model) and zoom-in on the $\mathrm{H}_{2} \mathrm{O}$ line at the same spectral resolution as in Fig. 1 to show that the line absorbs into the CMB (b, blue shading added for emphasis). The black curve is a fit to the spectrum. The red dashed curve is the best-fit radiative transfer model. 


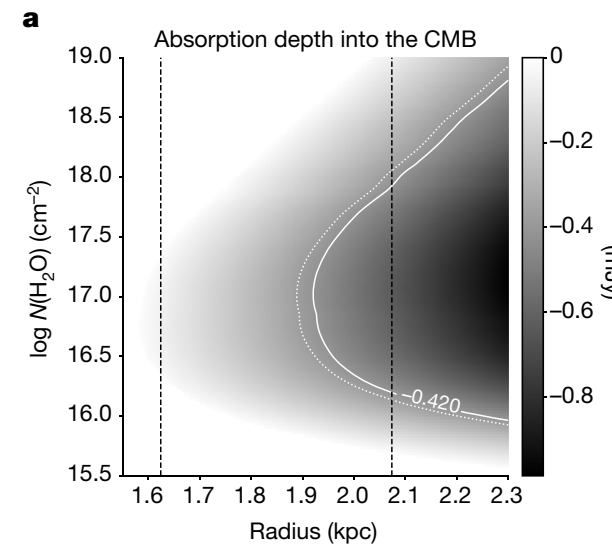

Fig. 3 | Radiative transfer models for HFLS3 and constraints on the CMB temperature. a, Model grid for the predicted line-absorption strength for $T_{\mathrm{CMB}}(z=6.34)=20.0 \mathrm{~K}$ (greyscale) as a function of $\mathrm{H}_{2} \mathrm{O}$ column density ( $y$ axis) and radius of the dust-emission region at $108 \mu \mathrm{m}$ ( $x$ axis). The white curves show the parameter space allowed by the measurement (solid line) and the $-1 \sigma$ r.m.s. uncertainty region (dotted line). The dashed black lines show the measured continuum size (left) and $+1 \sigma$ r.m.s. uncertainty region (right). The overlapping region between the white boundary (that is, the minimum allowed absorption strength) and the size measurement (that is, the minimum required emitting area at $100 \%$ covering fraction) is the allowed parameter space for the absorption strength within $1 \sigma$ r.m.s. The minimum required radius at $N\left(\mathrm{H}_{2} \mathrm{O}\right) \sim 10^{17} \mathrm{~cm}^{-2}$ is due to a minimum in $T_{\text {ex }}$ in the models. b, Constraints on $T_{\text {CMB }}$ for the observed absorption strength (green line and shaded region) at the

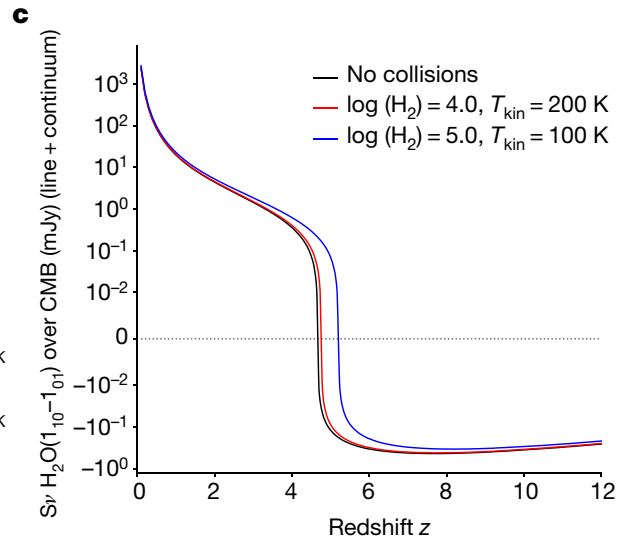

The over-proportional de-population of the $1_{10}$ level occurs because the $\mathrm{H}_{2} \mathrm{O}\left(2_{21}-1_{10}\right)$ transition at $108 \mu \mathrm{m}$ dominates the modification of the level population ${ }^{9,10}$. This transition lies near the peak of the dust spectral energy distribution, where the dust emission has a higher optical depth than at $538 \mu \mathrm{m}$, where the $\mathrm{H}_{2} \mathrm{O}\left(1_{10}-1_{01}\right)$ transition occurs. The increase in dust optical depth with wavelength leads to an increased availability of $108-\mu \mathrm{m}$ photons relative to $538-\mu \mathrm{m}$ photons compared with the thermal equilibrium case of a single-black-body radiation field. This implies that the $2_{21}-1_{10}$ transition at $108 \mu \mathrm{m}$ is exposed to a more intense infrared radiation field than the $1_{10}-1_{01}$ transition at $538 \mu \mathrm{m}$. For the infrared radiation field of HFLS3, the models therefore suggest that both $\mathrm{H}_{2} \mathrm{O}$ transitions should be found in absorption, but that the $2_{21}-1_{10}$ transition (which is not covered by our observations) will contribute a larger fraction to the $1_{10}$ level de-population than that found for the thermal equilibrium case. The CMB photons, on the other hand, only provide the base population expected for the thermal black-body equilibrium case. This causes a reduced excitation temperature in the $1_{10}-1_{01}$ transition compared with the thermal equilibrium case, unless the $1_{01}$ level is even more substantially de-populated due to the $\mathrm{H}_{2} \mathrm{O}\left(2_{12}-1_{01}\right)$ transition at $180 \mu \mathrm{m}$. This, however, does not occur, as our models show that this transition is expected to be seen in emission (at a line strength approximately $3-5$ times below a previously reported upper limit $^{5}$ ). This is because the upper-level population of the $2_{12}-1_{01}$ transition is also affected by the population of the $2_{12}$ level through the $2_{21}-2_{12}$ line, which appears in emission due to the pumping of its upper $2_{21}$ state by the $2_{21}-1_{10}$ absorption line. As such, the models suggest a net deficit in the upper-level population of the $1_{10}-1_{01}$ transition compared with the thermal equilibrium case, which causes the $T_{\mathrm{ex}}$ of the line to end up below the $\mathrm{CMB}$ temperature.

The RADEX models yield $T_{\mathrm{ex}}=17.4 \mathrm{~K}$ due to this level-population modification. To translate model-predicted temperature differences into an absorption-line flux that can be compared with the observations, the size of the emitting/absorbing region needs to be known. Based on NOEMA observations at rest-frame $122 \mu \mathrm{m}$ (Extended Data Fig. 2), we estimate the dust continuum size of the emitting region at
$108 \mu \mathrm{m}$ (the wavelength of the pumping transition) of HFLS3 to be $r_{108 \mu \mathrm{m}}=1.62 \pm 0.45$ kiloparsecs $(\mathrm{kpc})$. Within the uncertainties of the size estimate, the RADEX models suggest that the strength of the observed $\mathrm{H}_{2} \mathrm{O}$ absorption can be reproduced over about two orders of magnitude in $\mathrm{H}_{2} \mathrm{O}$ column density, with a lower limit of around $10^{16} \mathrm{~cm}^{-2}$. The minimum covering fraction of the dust continuum is about $60 \%$ when conservatively leaving $T_{\mathrm{CMB}}$ as a free parameter $(100 \%$ is assumed for the grid shown in Fig. 3a). The upper limit for the $\mathrm{H}_{2}$ column density implied by the gas mass of HFLS3 of $(1.04 \pm 0.09) \times 10^{11} \mathrm{M}_{\text {sun }}\left(\right.$ ref. $\left.^{5}\right)$ provides a lower limit to the gas phase $\left[\mathrm{H}_{2} \mathrm{O}\right] /\left[\mathrm{H}_{2}\right]$ abundance of $>2 \times 10^{-7}$, which falls within the range of $10^{-9}-10^{-5}$ found for nearby starbursts ${ }^{11}$. The small difference $\Delta T=T_{\mathrm{ex}}-T_{\mathrm{CMB}}=-2.6 \mathrm{~K}$ is, therefore, sufficient to explain the observed strength of the $\mathrm{H}_{2} \mathrm{O}\left(1_{10}-1_{01}\right)$ absorption line towards the CMB in HFLS3 when assuming a layer of cold, diffuse $\mathrm{H}_{2} \mathrm{O}$-bearing gas with a high covering fraction in front of the warm dust continuum source associated with the $\mathrm{H}_{2} \mathrm{O}$ emission lines.

As the absorption line is observed in contrast to the $\mathrm{CMB}$, we can use the strength of the absorption line to obtain a measurement of $T_{\mathrm{CMB}}$ at the redshift of HFLS3. The RADEX models suggest that, to detect the $\mathrm{H}_{2} \mathrm{O}\left(1_{10}-1_{01}\right)$ line in absorption against the CMB, $T_{\mathrm{CMB}}(z=6.34)$ must be $>7-8 \mathrm{~K}$, independent of the model assumptions (see Methods). The observed strength of the signal suggests $16.4 \mathrm{~K}<T_{\mathrm{CMB}}(z=6.34)<30.2 \mathrm{~K}$ $\left(1 \sigma\right.$, or $\left.12.8 \mathrm{~K}<T_{\mathrm{CMB}}(z=6.34)<34.0 \mathrm{~K} 2 \sigma\right)$ for HFLS3 when treating $T_{\text {dust }}$, $\beta_{\mathrm{IR}}$ and the wavelength where the dust optical depth reaches unity as free-fitting parameters for each dust continuum size sampled by the models. This explains why the effect has not been previously seen. $T_{\mathrm{CMB}}$ must be sufficiently high to satisfy the requirement of a notable $\mathrm{H}_{2} \mathrm{Ol}_{10}$ level population due to the $\mathrm{CMB}$, such that a de-population by the infrared radiation field of the starburst will lead to a sufficiently important decrement to be observable in absorption against the CMB. This limits observability to $z>4.5$ for dust spectral energy distribution shapes and dust continuum sizes of star-forming galaxies like HFLS3 (Fig. 3c), where only a few spectra at rest-frame $538 \mu \mathrm{m}$ with sufficient signal-to-noise ratio to detect the effect exist. This differs from molecules like $\mathrm{H}_{2} \mathrm{CO}$, for which absorption against the $\mathrm{CMB}$ has been predicted to occur at 


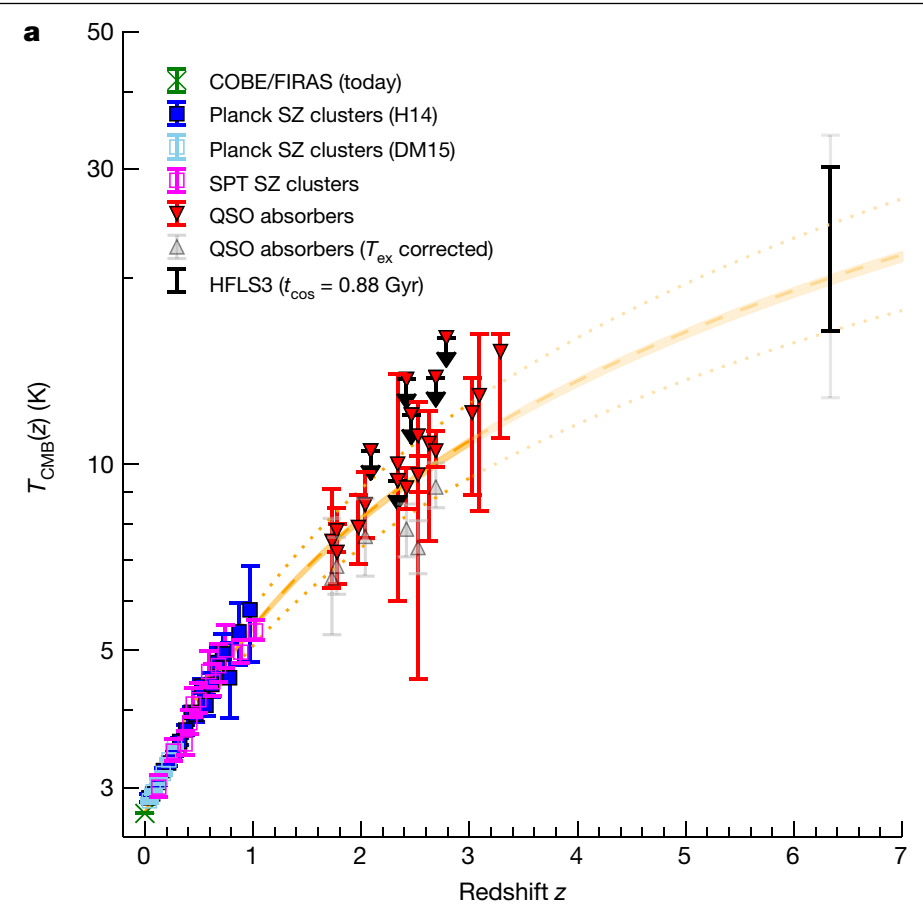

Fig. 4 | Measurements of the CMB temperature as a function of redshift ${ }^{3,17,19-28}$.a, $1 \sigma$ (black) and $2 \sigma$ r.m.s. (grey) uncertainties are shown for HFLS3 and $1 \sigma$ r.m.s. uncertainties elsewhere. b, Same data but dividing out the $(1+z)$ redshift scaling of the $\mathrm{CMB}$ expected from $\Lambda$ CDM. Previous direct measurements are from CMB mapping at $z=0$ and SZ effect measurements of galaxy clusters in contrast to the CMB out to $z \sim 1$ (left zoom-in panel in b). Additional measurements are from ultraviolet absorption systems along the lines of sights to quasars out to $z \sim 3$. The downward (upward) triangles are not corrected (corrected) for the contribution of collisional excitation in the diffuse interstellar medium to the excitation temperature $T_{\text {ex }}$ of the tracer (right zoom-in panel in b; green dots show an alternative proposed correction $\left.^{3}\right)^{21,27}$. The separation of these pairs of points for the same sources is an indication of the systematic uncertainties on top of the statistical uncertainties indicated by the error bars. $\mathrm{The}_{2} \mathrm{O}$-based measurement of

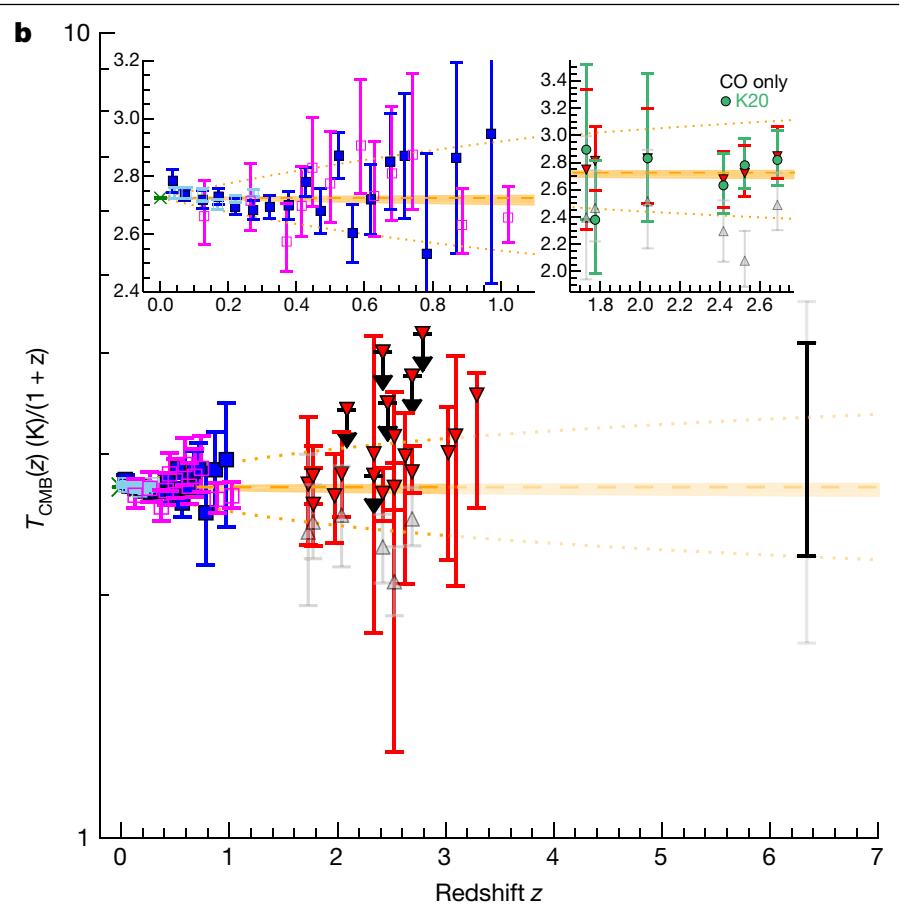

HFLS3, like those up to $z \sim 1$, is in contrast to the CMB, but-as a line measurement-it is more precise in redshift. It is not subject to the same uncertainties in $T_{\mathrm{ex}}$ as the intermediate redshift measurements, because collisions can only decrease (rather than boost) the resulting absorption strength into the $\mathrm{CMB}$ for the $\mathrm{H}_{2} \mathrm{O}$-based measurement. They are also unlikely to play an important role due to the high density required to collisionally excite the relevant $\mathrm{H}_{2} \mathrm{O}$ lines. Ignoring collisions results in the most conservative estimate of $T_{\mathrm{CMB}}$ for HFLS3. The orange shaded region shows a $T_{\mathrm{CMB}}=T_{\mathrm{CMB}}(z=0)^{*}(1+z)^{1-\beta}$ fit to the data in Extended Data Table 1 and its uncertainty (where $T_{\mathrm{CMB}}(z=0)=2.72548 \pm 0.00057 \mathrm{~K}\left(\right.$ ref. $\left.^{7}\right)$ and $\left.\beta=\left(3.4_{-7.3}^{+8.1}\right) \times 10^{-3}\right)$, the orange dashed line indicates the $\beta=0$ case corresponding to the standard cosmology and the dotted lines indicate $\mathrm{a} \pm 10 \%$ deviation in $1-\beta$.

lead to departures from this linear behaviour, including the evolution of physical constants ${ }^{14}$, decaying dark-energy models ${ }^{15}$ and axion-photon-like coupling processes ${ }^{16,17}$. Direct measurements of $T_{\mathrm{CMB}}$ are, thus, a crucial test of cosmology, but they are currently limited to $z<1$, due to the lack of sufficiently precise measurements of the thermal SZ effect in galaxy clusters at higher redshifts (Fig. 4; see Methods for further details). A limited sample of additional constraints exists at $z=1.8-3.3$ based on measurements of $T_{\mathrm{ex}}$ for the ultraviolet transitions of $\mathrm{CO},[\mathrm{Cl}]$ and $[\mathrm{CII}]$ in absorption-line systems along the lines of sight to quasars. These lines are not directly observed in contrast to the CMB, and they use the $T_{\mathrm{ex}}$ of these lines as a proxy of $T_{\mathrm{CMB}}$, such that the resulting $T_{\mathrm{CMB}}$ estimates are subject to model-dependent excitation corrections ${ }^{3,17-28}$. As an example, for the CO molecule, $T_{\mathrm{ex}}$ typically already exceeds $T_{\mathrm{CMB}}$ in the diffuse interstellar medium in the Milky Way due to collisional excitation, showing a rising excess with increasing $\mathrm{CO}$ optical depth due to photon trapping ${ }^{18}$. In contrast, our models suggest that collisional excitation of $\mathrm{H}_{2} \mathrm{O}$ becomes important only at very high densities, such that $\mathrm{H}_{2} \mathrm{O}$-based measurements are probably only minimally affected by this effect. The $\mathrm{H}_{2} \mathrm{O}$ absorption against the $\mathrm{CMB}$ at $z=6.34$ reported here thus provides the most direct constraint on $T_{\mathrm{CMB}}$ currently available at $z>1$. Indeed, the existence of this effect on its own directly implies that the $\mathrm{CMB}$ is warmer than at low redshift, because $T_{\mathrm{CMB}}$ must be sufficiently high to notably excite the $\mathrm{H}_{2} \mathrm{O}_{10}$ level, which lies $26.7 \mathrm{~K}$ above ground, as a basis for the observed decrement due to de-population of this level by the starburst radiation field. 


\section{Article}

A combined fit to the available data (Fig. 4) is consistent with the redshift scaling expected from $\Lambda$ CDM. Fitting for the adiabatic index $\gamma$ in the equation of state between pressure $P$ and energy density $\rho$ for the sum of baryonic and dark matter and radiation-that is, $P_{\mathrm{rm}}=(\gamma-1) \rho_{\mathrm{rm}}$ with a standard formalism (see Methods) - we find $\gamma=1.328_{-0.007}^{+0.008}$, which agrees with the standard value of $\gamma=4 / 3$ expected in $\Lambda$ CDM. At the same time, we find an effective dark energy equation of state parameter $w_{\text {eff }}=P_{\mathrm{de}} / \rho_{\mathrm{de}}=-1.011_{-0.017}^{+0.018}$, which is consistent with the $w=-1$ expectation for a dark energy density that does not evolve with time.

\section{Online content}

Any methods, additional references, Nature Research reporting summaries, source data, extended data, supplementary information, acknowledgements, peer review information; details of author contributions and competing interests; and statements of data and code availability are available at https://doi.org/10.1038/s41586-021-04294-5.

1. Sunyaev, R. A., \& Zel'dovich, I. A. B. Microwave background radiation as a probe of the contemporary structure and history of the universe. Annu. Rev. Astron. Astrophys. 18 537-560 (1980).

2. Rephaeli, Y. Comptonization of the cosmic microwave background: the Sunyaev-Zeldovich effect. Annu. Rev. Astron. Astrophys. 33, 541-580 (1995).

3. Klimenko, V. V. et al. Estimation of the cosmic microwave background temperature from atomic $\mathrm{Cl}$ and molecular $\mathrm{CO}$ lines in the interstellar medium of early galaxies. Astron. Lett. 46, 715-725 (2020).

4. Planck Collaboration. Planck 2018 results. I. Overview and the cosmological legacy of Planck. Astron. Astrophys. 641, A1 (2020).

5. Riechers, D. A. et al. A dust-obscured massive maximum-starburst galaxy at a redshift of 6.34. Nature 496, 329-333 (2013).

6. Riechers, D. A. et al. Rise of the titans: gas excitation and feedback in a binary hyper-luminous dusty starburst galaxy at $z \sim 6$. Astrophys. J. 907, 62 (2021).

7. Fixsen, D. J. The temperature of the cosmic microwave background. Astrophys. J. 707, 916 (2009).

8. van der Tak, F. F. S. et al. A computer program for fast non-LTE analysis of interstellar line spectra. With diagnostic plots to interpret observed line intensity ratios. Astron. Astrophys. 468, 627-635 (2007)

9. Gonzalez-Alfonso, E. et al. Herschel/PACS spectroscopy of NGC 4418 and Arp 22O: $\mathrm{H}_{2} \mathrm{O}$ $\mathrm{H}_{2}^{18} \mathrm{O}, \mathrm{OH},{ }^{18} \mathrm{OH}, \mathrm{OI}, \mathrm{HCN}$, and $\mathrm{NH}_{3}$. Astron. Astrophys. 541, A4 (2012).

10. Gonzalez-Alfonso, E. et al. Modeling the $\mathrm{H}_{2} \mathrm{O}$ submillimeter emission in extragalactic sources. Astron. Astrophys. 567, A91 (2014).

11. Liu, L. et al. HIFI spectroscopy of $\mathrm{H}_{2} \mathrm{O}$ submillimeter lines in nuclei of actively star-forming galaxies. Astrophys. J. 846, 5 (2017)

12. Darling, J. \& Zeiger, B. Formaldehyde silhouettes against the cosmic microwave background: a mass-limited, distance-independent, extinction-free tracer of star formation across the epoch of galaxy evolution. Astrophys. J. Lett. 749, L33 (2012).
13. Pavesi, R. et al. The CO Luminosity Density at High-z (COLDz) survey: a sensitive, large-area blind search for low-J $\mathrm{CO}$ emission from cold gas in the early universe with the Karl G. Jansky Very Large Array. Astrophys. J. 864, 49 (2018).

14. Uzan, J.-P. Varying constants, gravitation and cosmology. Living Rev. Relativ. 14, 2 (2011).

15. Jetzer, P. et al. Limits on decaying dark energy density models from the CMB temperature-redshift relation. Gen. Relativ. Gravitation 43, 1083-1093 (2011)

16. Jaeckel, J. \& Ringwald, A. The low-energy frontier of particle physics. Annu. Rev. Nucl. Part. Sci. 60, 405-437 (2010).

17. Hurier, G. et al. Measurement of the $T_{\mathrm{CMB}}$ evolution from the Sunyaev-Zel'dovich effect. Astron. Astrophys. 561, A143 (2014).

18. Burgh, E. B. et al. Direct measurement of the ratio of carbon monoxide to molecula hydrogen in the diffuse interstellar medium. Astrophys. J. 658, 446-454 (2007).

19. Songaila, A. et al. Measurement of the microwave background temperature at a redshift of 1.776. Nature 371, 43-45 (1994).

20. Ge, J., Bechtold, J., \& Black, J.H. A new measurement of the cosmic microwave background radiation temperature at $z=1.97$. Astrophys. J. 474, 67 (1997).

21. Srianand, R., Petitjean, P., \& Ledoux, C. The cosmic microwave background radiation temperature at a redshift of 2.34. Nature 408, 931-935 (2000).

22. Molaro, P. et al. The cosmic microwave background radiation temperature at $z_{\text {abs }}=3.025$ toward QSO 0347-3819. Astron. Astrophys. Lett. 381, L64-L67 (2002).

23. Noterdaeme, P. et al. The evolution of the cosmic microwave background temperature. Measurements of $T_{\mathrm{CMB}}$ at high redshift from carbon monoxide excitation. Astron. Astrophys. 526, L7 (2011).

24. Saro, A. et al. Constraints on the CMB temperature evolution using multiband measurements of the Sunyaev-Zel'dovich effect with the South Pole Telescope. Mon. Not. R. Astron. Soc. 440, 2610-2615 (2014).

25. de Martino, I. et al. Constraining the redshift evolution of the cosmic microwave background blackbody temperature with Planck data. Astrophys. J. 808, 128 (2015).

26. Avgoustidis, A. et al. Subpercent constraints on the cosmological temperature evolution. Phys. Rev. D 93, 043521 (2016).

27. Maeder, A. Scale-invariant cosmology and $\mathrm{CMB}$ temperatures as a function of redshifts. Astrophys. J. 847, 65 (2017)

28. Noterdaeme, P. et al. Discovery of a Perseus-like cloud in the early Universe. $\mathrm{HI}$-to- $\mathrm{H}_{2}$ transition, carbon monoxide and small dust grains at $z_{\text {abs }} \approx 2.53$ towards the quasar J0000+0048. Astron. Astrophys. 597, A82 (2017).

Publisher's note Springer Nature remains neutral with regard to jurisdictional claims in published maps and institutional affiliations.

Open Access This article is licensed under a Creative Commons Attribution 4.0 International License, which permits use, sharing, adaptation, distribution and reproduction in any medium or format, as long as you give appropriate credit to the original author(s) and the source, provide a link to the Creative Commons license, and indicate if changes were made. The images or other third party material in this article are included in the article's Creative Commons license, unless indicated otherwise in a credit line to the material. If material is not included in the article's Creative Commons license and your intended use is not permitted by statutory regulation or exceeds the permitted use, you will need to obtain permission directly from the copyright holder. To view a copy of this license visit http://creativecommons.org/licenses/by/4.0/.

c) The Author(s) 2022 


\section{Methods}

\section{NOEMA observations}

The target was observed in the $3-\mathrm{mm}$ wavelength band 1 (rest-frame $400 \mu \mathrm{m}$ ) with NOEMA as part of project S20DA (Principal Investigators: D. A. Riechers, F. Walter). Three partially overlapping spectral setups were observed under good weather conditions between 26July 2020 and 25 August 2020 with ten antennas in the most compact D configuration, using a bandwidth of $7.7 \mathrm{GHz}$ (dual polarization) at 2-MHz spectral resolution per sideband. We also included previously published $^{5}$ observations between 6 February 2012 and 31 May 2012 in the $A$ and $D$ configurations tuned to 110.128 and $113.819 \mathrm{GHz}$, respectively, and previously unpublished observations between 1 June 2012 and 4 June 2012 and on 10 July 2017 in the D configuration tuned to 78.544 and $101.819 \mathrm{GHz}$ taken as part of projects V0BD, W058, and S17CC (Principal Investigator:D. A. Riechers), all using $3.6 \mathrm{GHz}$ of bandwidth (dual polarization), yielding 21 observing runs in total. Nearby radio quasars were used for complex gain, bandpass and absolute flux calibration. The target was also observed in the $0.87-\mathrm{mm}$ wavelength band 4 (rest-frame $122 \mu \mathrm{m}$ ) with NOEMA as part of project XOCC (Principal Investigator: D. A. Riechers). Observations were carried out during three observing runs with six antennas in the A and C configurations under good weather conditions between 4 December 2013 and 12 March 2015, with the band 4 receivers tuned to $335.5 \mathrm{GHz}$ and using a bandwidth of $3.6 \mathrm{GHz}$ (dual polarization). Nearby radio quasars were used for complex gain, bandpass and absolute flux calibration. The GILDAS package was used for data calibration and imaging. All 3-mm data were combined to a single visibility cube before imaging. Imaging was carried out with natural baseline weighting. The band 4 data were also imaged with Briggs robust weighting to increase the spatial resolution. A map of the continuum emission at the frequency of the $\mathrm{H}_{2} \mathrm{O}$ line was created by averaging the visibility data over a bandwidth of $2.04 \mathrm{GHz}$ centred on the line. This range was chosen to avoid other lines in the bandpass. Continuum emission was subtracted from the $\mathrm{H}_{2} \mathrm{O}$ line cube in the visibility plane. Moment $\mathrm{O}$ images of the line absorption were created before and after continuum subtraction by integrating the signal over a bandwidth of $100 \mathrm{MHz}$, corresponding to $395 \mathrm{~km} \mathrm{~s}^{-1}$. The resulting r.m.s. noise levels are provided in Extended Data Fig. 2. We also make use of previously published ${ }^{5}$ rest-frame $158-\mu \mathrm{m}$ NOEMA data, which were adopted without further modification.

\section{Line and continuum parameters}

The flux of the $\mathrm{H}_{2} \mathrm{O}\left(1_{10}-1_{01}\right)$ line was extracted by simultaneous Gaussian fitting of the line and continuum emission (including a linear term for the continuum) in the one-dimensional spectrum shown in Fig. 1, which was extracted from the image cube. The source is unresolved at the frequency of the $\mathrm{H}_{2} \mathrm{O}\left(\mathrm{1}_{10}-\mathbf{1}_{01}\right)$ line, such that the main uncertainties are due to the slope of the continuum emission and the appropriate fitting of other nearby lines, in particular, $\mathrm{CO}(5-4)$. The uncertainties in these parameters are part of the quoted uncertainties. We find a line peak flux of $-818 \pm 145 \mu \mathrm{Jy}$ at a line full width half maximum (FWHM) of $507 \pm 111 \mathrm{~km} \mathrm{~s}^{-1}$, centred at a frequency of $75.8948 \mathrm{GHz}\left( \pm 46 \mathrm{~km} \mathrm{~s}^{-1}\right.$; the calibration uncertainties on the line FWHM and centre frequency are negligible and that on the line peak flux is $<10 \%$-that is, minor compared with the measurement uncertainty). Given the rest frequency of the line of $556.9359877 \mathrm{GHz}$, this corresponds to a redshift of 6.3383, which is consistent with the systemic redshift of HFLS3 $(z=6.3335$ and 6.3427 with uncertainties of \pm 14 and $\pm 54 \mathrm{~km} \mathrm{~s}^{-1}$ at Gaussian FWHM of $243 \pm 39$ and $760 \pm 152 \mathrm{~km} \mathrm{~s}^{-1}$, respectively, for the two velocity components detected in the $158-\mu \mathrm{m}$ [CII] line $)^{5}$. For comparison, the $\mathrm{H}_{2} \mathrm{O}\left(2_{02}-1_{11}\right)$ and $\mathrm{H}_{2} \mathrm{O}\left(2_{11}-2_{02}\right)$ emission lines in HFLS3 have FWHM of $805 \pm 129$ and $927 \pm 330 \mathrm{~km} \mathrm{~s}^{-1}$, respectively ${ }^{5}$-that is, only marginally broader than the $1_{10}-1_{01}$ line at the current measurement uncertainties. The continuum flux at the line frequency is $396 \pm 15 \mu \mathrm{Jy}$, corresponding to $48 \% \pm 9 \%$ of the absorption-line flux (the relative flux calibration uncertainty between the line and continuum emission is negligible). We also measured the $335.5-\mathrm{GHz}$ continuum flux by two-dimensional fitting to the continuum emission in the visibility plane. We find a flux of $33.9 \pm 1.1 \mathrm{mJy}$, which agrees with previous lower-resolution observations at the same wavelength ${ }^{5}$. The major (minor) axis FWHM diameter of the source is $0.617 \pm 0.074 \operatorname{arcsec}(0.37 \pm 0.20 \operatorname{arcsec})$. This yields the physical source size quoted in the main text at the redshift of HFLS3.

\section{Brightness temperature contrast}

The $\mathrm{H}_{2} \mathrm{O}\left(1_{10}-1_{01}\right)$ line leads to a decrement in continuum photons from the starburst and, as such, is observed as a lack of continuum emission at its frequency at the position of the starburst. It therefore appears as negative flux in an image where starburst continuum emission has been subtracted. In addition, (sub)millimetre-wavelength interferometric images reveal structure against a flat sky background defined by the large-scale CMB surface brightness, which the interferometer does not detect itself due to its limited spatial sampling. Therefore the fraction of the signal due to the decrement in $\mathrm{CMB}$ photons at the position of the starburst not only appears as negative flux without subtracting any further signal but it also corresponds to a lack of continuum emission at the line frequency in practice. As the mere presence of an absorption-line signal stronger than the measured continuum emission implies absorption against the $\mathrm{CMB}$, this interpretation is not limited by uncertainties in the galaxy continuum flux or uncertainties in the absolute flux calibration.

\section{Line-excitation modelling}

RADEX is a radiative transfer program to analyse interstellar line spectra by calculating the intensities of atomic and molecular lines, assuming statistical equilibrium and considering collisional and radiative processes, as well as radiation from background sources. Optical depth effects are treated with an escape probability method ${ }^{8}$. Studies of nearby star-forming galaxies show that the observed absorption strengths of the ground-state $\mathrm{H}_{2} \mathrm{O}$ and $\mathrm{H}_{2} \mathrm{O}^{+}$transitions are due to cooler gas that is located in front of, and irradiated by, a warmer background source that is emitting the infrared continuum light that also excites the higher-level $\mathrm{H}_{2} \mathrm{O}$ emission lines ${ }^{11,29}$. We therefore adopt the same geometry for the modelling in this work, which is adequately treated within RADEX (that is, treating the dust continuum plus the CMB as background fields for the absorbing material $)^{8}$. The dust continuum emission is modelled as a grey body with treating $T_{\text {dust }}, \beta_{\mathrm{IR}}$ and the wavelength where the dust optical depth reaches unity as free-fitting parameters for each dust continuum size and $T_{\mathrm{CMB}}$ sampled by the models. The observed spectral energy distribution of HFLS3, including all literature ${ }^{5}$ photometry and the measurements presented in this work, is then treated as the contrast between the dust continuum and CMB background fields, such that the resulting fit parameters for the dust continuum source change with $T_{\mathrm{CMB}}$ in a self-consistent manner. In the RADEX models, we derive the $\mathrm{H}_{2} \mathrm{O}$ peak absorption depth into the CMB. We then multiply the best matching peak absorption depth found by RADEX with a Gaussian matched to the fitted line centroid and line width obtained from the observed line profile in Fig. 2 to determine the model line profile. In this approach, the shallower absorption in the line wings either corresponds to a lower filling factor of the $\mathrm{H}_{2} \mathrm{O}$ layer at the corresponding velocities or to lower $\mathrm{H}_{2} \mathrm{O}$ column densities. Although collisions of $\mathrm{H}_{2} \mathrm{O}$ molecules with $\mathrm{H}_{2}$ is another mechanism that can modify the level populations especially at very high gas densities (which is an important mechanism for the cooling of low-excitation-temperature transitions of molecules like $\mathrm{H}_{2} \mathrm{CO}$ to below $\left.T_{\mathrm{CMB}}\right)^{12,30}$, the RADEX models show that they do not affect our findings (see Fig. 3c). We therefore adopt models with essentially no collisions by assuming a very low gas density of $n\left(\mathrm{H}_{2}\right)=10 \mathrm{~cm}^{-3}$. We then compare our findings to those obtained when adopting conditions that are similar to those found in local starburst galaxies ${ }^{11}$ and to those found for high-density environments with $n\left(\mathrm{H}_{2}\right)>10^{5} \mathrm{~cm}^{-3}$. The cross sections 
for collisions out of the $1_{01}$ level are always larger than those out of the $1_{10}$ level, independent of the collision partner and the temperature at which the collisions take place ${ }^{31-33}$. Therefore collisions cannot be responsible for an over-proportional de-population of the $1_{10}$ level relative to the $1_{01}$ ground state, and the net effect of including collisions is a decrease in the absorption depth into the $\mathrm{CMB}$ by reducing the $T_{\mathrm{CMB}}-T_{\mathrm{ex}}$ temperature difference at very high gas densities compared with cases without collisions. For reference, the effect of collisions on the determination of $T_{\mathrm{CMB}}$ is negligible for the typical conditions found in local starbursts (that is, $\left.n\left(\mathrm{H}_{2}\right) \sim 10^{4} \mathrm{~cm}^{-3} ; T_{\text {kin }}=20-180 \mathrm{~K}\right)^{11}$ and only starts to have an impact for very high densities $n\left(\mathrm{H}_{2}\right)>10^{5} \mathrm{~cm}^{-3}$. For a given continuum source size, the constraints on $T_{\mathrm{CMB}}$ would therefore be tighter (that is, would more quickly become inconsistent with the observations) for the high-density case than for the case without collisions, such that the latter approach is more conservative (see Fig. 3b). The overall impact of collisional excitation would therefore be more stringent requirements on the source size, covering fraction and water column, such that their inclusion would only further strengthen our conclusions. We note that this is the opposite effect to the case of the studies of ultraviolet lines ${ }^{3,17,19-28}$, where neglecting collisional excitation results in less conservative constraints on $T_{\mathrm{CMB}}$. If we were to assume that the $\mathrm{H}_{2} \mathrm{O}$ absorption were to emerge from within the infrared continuum-emitting region, a larger source size would probably be required to obtain the same absorption-line strength due to a reduced effective radiation field strength from the starburst. Previous modelling attempts of nearby galaxies assuming such geometries have not been able to produce $\mathrm{H}_{2} \mathrm{O}\left(1_{10}-1_{01}\right)$ line absorption on the scales necessary to explain the observations of HFLS3, which may indicate that even more complex assumptions would be required ${ }^{11}$. Thus, the resulting constraints would, once again, be less conservative, perhaps acting in a similar manner as the high-density case. Excluding both of these effects from the models leads to a maximally conservative estimate of $T_{\mathrm{CMB}}$ and its uncertainties. Assuming a plane-parallel or similar geometry instead of a spherical geometry would only have a minor impact on our findings ${ }^{8}$. The models shown in Fig. 3 assume a filling factor of unity, which is the most conservative possible assumption. A more clumpy geometry with a lower covering fraction remains possible for all $T_{\mathrm{CMB}}$ values for which the predicted absorption strength exceeds the observed value (see shaded regions in Fig. 3b). For reference, the minimum covering fractions consistent with the continuum size at the observed signal strength are shown for the different cases considered in Fig. 3. The line absorption is also found to be optically thick, with an optical depth of $\tau_{\mathrm{H} 2 \mathrm{O}}=21.1$ for the solution shown in Fig. 2b. To determine the redshift above which the effect becomes observable (Fig. $3 \mathrm{c}$ ), we fixed $r_{108 \mu \mathrm{m}}, T_{\text {dust }}, \beta_{\mathrm{IR}}$ and $M_{\text {dust }}$ to the observed values and the $\mathrm{H}_{2} \mathrm{O}$ column density to the value corresponding to the model spectrum. $\mathrm{H}_{2} \mathrm{O}$ line absorption into the dust continuum of HFLS3 would already become visible at $z>2.9$, but absorption into the CMB only becomes observable at $z>4.5$ (or higher for $\mathrm{H}_{2}$ densities of $>10^{5} \mathrm{~cm}^{-3}$ ). These values account for changes in the shape of the dust grey-body spectrum (that is, changes in the relative availability of $538-\mu \mathrm{m}$ and $108-\mu \mathrm{m}$ photons) due to changes in $T_{\mathrm{CMB}}$ with redshift. To better quantify the impact of different modelling parameters, we have varied $T_{\text {dust }}$ and $\beta_{\mathrm{IR}}$ beyond their previously estimated uncertainties (nominal reference values without considering variations in $T_{\mathrm{CMB}}$ from the literature are $T_{\text {dust }}=63.3_{-5.8}^{+5.4} \mathrm{~K}$ and $\left.\beta_{\mathrm{IR}}=1.94_{-0.09}^{+0.07}\right)^{5,6}$. This is necessary because both parameters are dependent on the varying $T_{\mathrm{CMB}}$ in our models (and therefore are changing parameters in Fig. 3b, c), such that their true uncertainties need to be re-evaluated. We independently varied $\beta_{\mathrm{IR}}$ in the 1.6-2.4 range and $T_{\text {dust }}$ in the $\pm 20 \mathrm{~K}$ range as functions of $T_{\mathrm{CMB}}$ around the best-fit values. This shows that $\beta_{\mathrm{IR}}>2.0$ and $T_{\text {dust }}$ lower by more than $10 \mathrm{~K}$ from the best fits yield very poor fits to the spectral energy distribution data, whereas $\Delta \beta_{\mathrm{IR}}>-0.1$ below the best-fit value would require a larger continuum size than the measured $r_{108 \mathrm{~mm}}+1 \sigma$ and therefore are disfavoured by the size constraint. Excluding these ranges, the extrema across this entire range would extend the uncertainty range in the predicted $T_{\mathrm{CMB}}$ by only -1.7 and $+5.4 \mathrm{~K}$ and -0.8 and $+4.4 \mathrm{~K}$ for the $r_{108 \mu \mathrm{m}}+1 \sigma$ and $r_{108 \mu \mathrm{m}}+2 \sigma$ cases, respectively. For comparison, the difference between the $+1 \sigma$ and $+2 \sigma$ uncertainty ranges is -3.6 and $+3.8 \mathrm{~K}$ ). This shows that the impact of the uncertainties in the dust spectral energy distribution fitting parameters on those in $T_{\mathrm{CMB}}$ are subdominant to those in the continuum size measurement. Conversely, we have studied the impact of changes in $T_{\mathrm{CMB}}$ on the best-fit $T_{\text {dust }}$ and $\beta_{\mathrm{IR}}$. For the values corresponding to $r_{108 \mu \mathrm{m}}+1 \sigma$ and $r_{108 \mu \mathrm{m}}+2 \sigma$ ranges, $T_{\text {dust }}$ typically changes by $<0.5 \mathrm{~K}$ and $\beta_{\mathrm{IR}}$ typically changes by $<0.1-0.2$ when varying the parameters independently. These changes are larger than the actual uncertainties, because the fit to the dust spectral energy distribution becomes increasingly poorer with these single-parameter variations. At the same time, these changes are subdominant to those induced by changes in dust continuum size within the $+1 \sigma$ and $+2 \sigma$ uncertainty ranges, which is consistent with our other findings.

\section{Other $\mathrm{H}_{2} \mathrm{O}$ transitions in HFLS3}

Five $\mathrm{H}_{2} \mathrm{O}$ lines were previously detected towards HFLS3 $\left(2_{02}-1_{11}\right.$, $2_{11}-2_{02}, 3_{12}-2_{21}, 3_{12}-3_{03}$ and $3_{21}-3_{12}$ ) and two additional lines were tentatively detected $\left(4_{13}-4_{04} \text { and } 4_{22}-4_{13}\right)^{5}$. The $J_{\text {up }}=3$ transitions are due to ortho- $\mathrm{H}_{2} \mathrm{O}$ and all other transitions are due to para- $\mathrm{H}_{2} \mathrm{O}$. All of these transitions appear in emission. Given the high critical densities of these transitions, our RADEX models cannot reproduce the strength of these lines as the same time as the observed ortho- $\mathrm{H}_{2} \mathrm{O}\left(1_{10}-1_{01}\right)$ absorption strength, which suggests that they emerge from different gas components. For reference, to reproduce the strength of the $\mathrm{H}_{2} \mathrm{O}\left(2_{11}-2_{02}\right)$ in Fig. 1 alone with collisional excitation, $n\left(\mathrm{H}_{2}\right)=2 \times 10^{7} \mathrm{~cm}^{-3}$ and $T_{\text {kin }}=200 \mathrm{~K}$ would be required, but the $\mathrm{H}_{2} \mathrm{O}\left(1_{10}-1_{01}\right)$ would no longer appear in absorption against the $\mathrm{CMB}$ if it were to emerge from the same gas component. This is consistent with the picture that the $\mathrm{H}_{2} \mathrm{O}$ absorption is due to a cold gas component along the line of sight to the warm gas that gives rise to the emission lines ${ }^{11}$. Observations of the para- $\mathrm{H}_{2} \mathrm{O}\left(1_{11}-\mathrm{O}_{00}\right)$ ground state do not currently exist for HFLS3, but our models do not show this line in absorption towards the CMB.

\section{Origin of the lower and upper limits on $T_{\mathrm{CMB}}$}

Our models show that the lower limit on $T_{\mathrm{CMB}}$ at a given redshift based on the observed $\mathrm{H}_{2} \mathrm{O}$ absorption is due to the minimum 'seed' level population due to the $\mathrm{CMB}$ black-body radiation field. To determine a conservative lower limit, we have calculated models with continuum sizes up to $r_{108 \mu \mathrm{m}}=5 \mathrm{kpc}$ (see Fig. $3 \mathrm{~b}$ ), corresponding to $\mathrm{a}+7.5 \sigma$ deviation from the observed continuum size, and recorded the temperatures at which such weakly constrained models turn into absorption. We find that this results in a lower limit of $T_{\mathrm{CMB}}>7-8 \mathrm{~K}$, independent of the model assumptions. This finding alone does not explain the existence of an upper limit in Fig. 3b. For a given size of the dust continuum emission, an increase in $T_{\mathrm{CMB}}$ also requires an increase in $M_{\text {dust }}$ to still reproduce the observed dust spectral energy distribution, which leads to an effective increase in the dust optical depth at a given wavelength. The result of a rising optical depth is that the grey-body spectrum between 538 and $108 \mu \mathrm{m}$ increasingly resembles a black-body spectrum and, hence, a decrease in the $\mathrm{H}_{2} \mathrm{O}$ absorption against the CMB. This effect is responsible for the upper limit in allowed $T_{\mathrm{CMB}}$ for a given dust continuum size and absorption strength.

\section{Uncertainties of $T_{\mathrm{CMB}}$ measurements}

The uncertainties shown for the literature data in Fig. 4 are adopted from the literature sources without modification, and they typically represent the statistical uncertainties from the individual measurements or sample averages. Individual cluster measurements of the thermal SZ effect may be affected by dust associated with foreground galaxies or the Milky Way, the galaxy clusters or background galaxies that may be amplified by gravitational lensing, uncertainties in the reconstruction of the Compton- $y$ parameter maps due to flux 
uncertainties, radio emission due to active galactic nuclei and/or relics, the kinetic and relativistic SZ effects, and general bandpass and calibration uncertainties ${ }^{17}$. Furthermore, uncertainties on the cluster geometry-and therefore line-of-sight travel distance of the CMB photons through the cluster-and on the temperature of the intra-cluster gas limit the precision of individual SZ measurements. Sample averages may also be affected by systematics in the stacking procedures. Individual data points deviate by up to at least two standard deviations from the trend, which may indicate residual uncertainties beyond the statistical error bars provided, such that the error bars shown in Fig. 4 are underestimated. The main source of uncertainty for the ultraviolet absorption-line-based measurements are due to the assumption of no collisional excitation, which is not taken into account in the statistical uncertainties shown in Fig. 4. Attempts to take this effect into account appear to suggest substantially larger uncertainties than indicated by individual error bars ${ }^{27}$ (Fig. 4). To expand on earlier estimates ${ }^{21}$, we have calculated RADEX models for typical $T_{\text {kin }}, n(\mathrm{H})$ and column densities found from $[\mathrm{CI}]$ measurements in the diffuse interstellar medium $^{34}$, which suggests that collisional excitation contributes to the predicted $T_{\mathrm{ex}}$ of the lower fine-structure transition. Although we show the original unmodified data, the ultraviolet-based measurements are therefore subject to uncertainties due to model-dependent excitation corrections in addition to the statistical uncertainties. Furthermore, the fine-structure levels of tracers like the $[\mathrm{CI}]$ lines can be excited by ultraviolet excitation and following cascades. To constrain $T_{\mathrm{CMB}}$ based on these measurements, the kinetic temperature, particle density and local ultraviolet radiation field must be known, and are typically determined based on tracers other than the species used to constrain $T_{\mathrm{CMB}}$. Also, some measurements are based on spectrally unresolved lines, which limits the precision of kinetic temperature measurements based on thermal broadening ${ }^{21}$. Owing to these uncertainties, the ultraviolet absorption-line-based measurements are probably consistent with the standard $\Lambda C D M$ value, but they do not constitute a direct measurement of $T_{\mathrm{CMB}}$ without notable further assumptions. For reference, the median $T_{\mathrm{CMB}} /(1+z)$ estimate based on the [CI] measurements alone (excluding upper limits) is $3.07 \mathrm{~K}$, with a median absolute deviation of $0.09 \mathrm{~K}$ and a standard deviation of $0.31 \mathrm{~K}$. Therefore the current sample median deviates from the $\Lambda \mathrm{CDM}$ value by about one standard deviation. A combination of the (uncorrected) measurements based on $\mathrm{CO},[\mathrm{CI}]$ and $[\mathrm{CII}]$ provides a median value of $2.84 \mathrm{~K}$, with a median absolute deviation of $0.15 \mathrm{~K}$ and a standard deviation of $0.25 \mathrm{~K}$. This highlights the importance of the corrections discussed above and in the literature and the value of measurements with systematic uncertainties that differ from this method to obtain a more complete picture. The main source of uncertainty of the $\mathrm{H}_{2} \mathrm{O}$-based measurements, beyond the caveats stated in the line-excitation-modelling section, are the statistical uncertainties on the source size, the lack of a direct measurement of the absorbing $\mathrm{H}_{2} \mathrm{O}$ column density, variations in the dust mass absorption coefficient and the filling factor. Given the high metallicity suggested by other molecular line detections, the limitation to high filling factors due to the source size and the constraint on the gas mass from dynamical mass measurements, the main source of uncertainty resides in the source size due to limited spatial resolution in the current data. As such, major improvements should be possible by obtaining higher, (sub-)kpc resolution (that is, $<0.2$ ") imaging with the Atacama Large Millimeter/submillimeter Array (ALMA; for other targets) and planned upgrades to NOEMA, and, in the future, with the Next Generation Very Large Array (ngVLA). Statistical uncertainties will also be greatly reduced by observing larger samples of massive star-forming galaxies over the entire redshift range where measurements are possible, closing the gap to SZ-based studies, which are currently limited to $z<1$. The resulting improvement in precision will provide the constraints that are necessary to confirm or challenge the evolution of the CMB temperature with redshift predicted by standard cosmological models.

\section{Accessibility of the line signal}

The frequency range currently covered by NOEMA is 70.4-119.3, 127.0-182.9 and 196.1-276.0 GHz (with greatly reduced sensitivity above about 115 and $180 \mathrm{GHz}$ in the first two frequency ranges). ALMA covers the $84-500-\mathrm{GHz}$ range with gaps at $116-125$ and $373-385 \mathrm{GHz}$, with a future extension down to $65 \mathrm{GHz}$ (with greatly reduced sensitivity below about $67 \mathrm{GHz}$ ). The ngVLA is envisioned to cover the 70-116-GHz range. Excluding regions of poor atmospheric transparency, the $\mathrm{H}_{2} \mathrm{O}\left(1_{10}-1_{01}\right)$ line is therefore observable in these frequency ranges at redshifts of $z=0.1-0.4,0.5-2.0,2.1-3.4$ and 3.8-6.9 in principle, but the detectability of the line in absorption against the CMB is probably limited to the $z \sim 4.5-6.9$ range if the spectral energy distribution shape of HFLS3 is representative. At lower frequencies, the Karl G. Jansky Very Large Array and, in the future, ALMA and the ngVLA also provide access to the $<52-\mathrm{GHz}$ range, such that the signal also becomes observable at $z>9.7$ in principle. In conclusion, the absorption of the ground-state $\mathrm{H}_{2} \mathrm{O}$ transition against the CMB identified here could be traced from the ground towards star-forming galaxies across most of the first approximately 1.5 billion years of cosmic history.

\section{Detectability of the line signal for different spectral energy distribution shapes}

To investigate whether the effect is expected to be detectable for different galaxy populations, we have applied our modelling to the $z=3.9$ quasar APM 08279+5255, for which the dust spectral energy distribution is composed of a dominant $220-\mathrm{K}$ dust component and a weaker 65-K dust component, contributing only $10-15 \%$ to the far-infrared luminosity ${ }^{35-46}$. The models suggest that the line is expected to occur in emission and that it would not be expected to be detectable in absorption at any redshift out to at least $z=12$ in galaxies with similar dust spectral energy distributions. Other far-infrared-luminous, high-redshift, active galactic nucleus host galaxies typically show a stronger relative contribution of their lower-temperature dust components, such that the effect may remain detectable in less extreme cases. For galaxies with lower dust temperatures than HFLS3, the effect may be present even at lower redshifts, but is typically expected to be weaker in general and to disappear at redshifts where $T_{\mathrm{CMB}}$ approaches their $T_{\text {dust }}$. For a dust spectral energy distribution shape resembling the central region of the Milky Way but otherwise similar properties, the effect is expected to be reduced by more than two orders of magnitude at its redshift peak, and to become virtually unobservable at the redshift of HFLS3. Thus, dusty starburst galaxies appear to be some of the best environments to detect the effect.

\section{Derivation of equation of state parameters}

To determine the adiabatic index, we assume a standard FriedmannLemaitre-Robertson-Walker cosmology with zero curvature and a matter-radiation fluid that follows the standard adiabatic equation of state quoted in the main text. This would correspond to a redshift scaling $T_{\mathrm{CMB}}(z)=T_{\mathrm{CMB}}(z=0)^{*}(1+z)^{3(\gamma-1)}$ in the presence of a dark energy density that does not scale with redshift. The dark energy density is parameterized to scale with a power law $(1+z)^{m}$, where $m=0$ corresponds to a cosmological constant. With standard assumptions, this yields a redshift scaling of $T_{\mathrm{CMB}}\left(\right.$ ref. $\left.^{15}\right)$ :

$$
T_{C M B}(z)=T_{C M B}(z=0)(1+z)^{3(\gamma-1)}\left[\frac{\left(m-3 \Omega_{m, 0}\right)+m(1+z)^{(m-3)}\left(\Omega_{m, 0}-1\right)}{(m-3) \Omega_{m, 0}}\right]^{(\gamma-1)}
$$

and an effective dark energy equation of state $P_{\mathrm{de}}=w_{\text {eff }} \rho_{\mathrm{de}}$, where the effective equation of state parameter $w_{\text {eff }}=(m / 3)-1$. This fitting function is used here with a canonical value of $\Omega_{\mathrm{m}, 0}=0.315$ (ref. ${ }^{4}$ ). The uncertainty of $\Omega_{\mathrm{m}, 0}$ is small compared with all other sources of uncertainty 


\section{Article}

and, hence, is neglected. All data used in the fitting are provided in Extended Data Table 1 (refs. ${ }^{36-46}$ ).

\section{Data availability}

The spectral line data and model generated and analysed during this study as shown in Fig. 2 are linked to this manuscript in spreadsheet form. Additional versions of the NOEMA datasets (visibilities, images and spectra) are available from the corresponding author (D.A.R.) on reasonable request. All data are also available in the IRAM Science Data Archive (isda@iram.fr) under project IDs V0BD, W058, X0CC, S17CC, and S20DA. Source data are provided with this paper.

\section{Code availability}

The RADEX code used for the modelling presented in this work and shown in Fig. 3 is available at https://home.strw.leidenuniv.nl/ moldata/ radex.html.

29. Weiss, A. et al. HIFI spectroscopy of low-level water transitions in M 82. Astron. Astrophys. 521, L1 (2010).

30. Townes, C. H. Astronomical masers and lasers. Quantum Electron. 271031 (1997)

31. Mueller, H. S. P. et al. The Cologne database for molecular spectroscopy, CDMS. Astron. Astrophys. 370, L49-L52 (2001).

32. Dubernet, M.-L. et al. Rotational excitation of ortho- $\mathrm{H}_{2} \mathrm{O}$ by para- $\mathrm{H}_{2}\left(\mathrm{j}_{2}=\mathrm{O}, 2,4,6,8\right)$ at high temperature. Astron. Astrophys. 497, 911-925 (2009)

33. Daniel, F. et al. Rotational excitation of 45 levels of ortho/para- $\mathrm{H}_{2} \mathrm{O}$ by excited ortho/ para- $\mathrm{H}_{2}$ from $5 \mathrm{~K}$ to $1500 \mathrm{~K}$ : state-to-state, effective, and thermalized rate coefficients. Astron. Astrophys. 536, A76 (2011).

34. Jenkins, E. B., \& Tripp, T. M. The distribution of thermal pressures in the diffuse, cold neutral medium of our galaxy. II. An expanded survey of interstellar $\mathrm{CI}$ fine-structure excitations. Astrophys. J. 734, 65 (2011).

35. Weiss, A. et al. Highly-excited CO emission in APM 08279+5255 at $z=3.9$. Astron. Astrophys. 467, 955-969 (2007).

36. Cui, J. et al. Molecular hydrogen in the damped Lya absorber of Q1331+170. Astrophys. J. 633, 649 (2005)

37. Ledoux, C., Petitjean, P., \& Srianand, R. The Very Large Telescope Ultraviolet and Visible Echelle Spectrograph survey for molecular hydrogen in high-redshift damped Lyman a systems. Mon. Not. R. Astron. Soc. 346, 209-228 (2003).
38. Balashev, S. A. et al. Partial coverage of the broad-line region of Q1232+082 by an intervening $\mathrm{H}_{2}$-bearing cloud. Mon. Not. R. Astron. Soc. 418, 357-369 (2011)

39. Srianand, R. et al. First detection of $\mathrm{CO}$ in a high-redshift damped Lyman-a system. Astron. Astrophys. Lett. 482, L39-L42 (2008).

40. Ranjan, A. et al. Molecular gas and star formation in an absorption-selected galaxy: hitting the bull's eye at $z \simeq 2.46$. Astron. Astrophys. 618, A184 (2018).

41. Noterdaeme, P. et al. Spotting high-z molecular absorbers using neutral carbon: results from a complete spectroscopic survey with the VLT. Astron. Astrophys. 612, A58 (2018).

42. Balashev, S. A., Ivanchik, A. V., \& Varshalovich, D. A. HD/ $\mathrm{H}_{2}$ molecular clouds in the early Universe: the problem of primordial deuterium. Astron. Lett. 36, 761-772 (2010).

43. Noterdaeme, P. et al. A translucent interstellar cloud at $z=2.69: \mathrm{CO}, \mathrm{H}_{2}$, and $\mathrm{HD}$ in the line-of-sight to SDSS J123714.60 + 064759.5. Astron. Astrophys. 523, A80 (2010).

44. Balashev, S. A. et al. CO-dark molecular gas at high redshift: very large $\mathrm{H}_{2}$ content and high pressure in a low-metallicity damped Lyman alpha system. Mon. Not. R. Astron. Soc. 470, 2890-2910 (2017).

45. Jorgenson, R. A., Wolfe, A. M., \& Prochaska, J. X. Understanding physical conditions in high-redshift galaxies through $\mathrm{C}$ I fine structure lines: data and methodology. Astrophys. J. 722, 460 (2010).

46. Guimaraes, R. et al. Metallicities, dust, and molecular content of a QSO-damped Lya system reaching $\log N(\mathrm{HI})=22$ : an analog to GRB-DLAs. Astron. J 143, 147 (2012).

Acknowledgements D.A.R. acknowledges support from the National Science Foundation under grant numbers AST-1614213 and AST-1910107. D.A.R. also acknowledges support from the Alexander von Humboldt Foundation through a Humboldt Research Fellowship for Experienced Researchers. This work is based on observations carried out under project numbers VOBD, W058, XOCC, S17CC, and S2ODA with the IRAM NOEMA interferometer. IRAM is supported by INSU/CNRS (France), MPG (Germany) and IGN (Spain)

Author contributions D.A.R. led the project and writing of the manuscript. A.W. produced the models used in this work. F.W. was a co-Principal Investigator of the main observing proposal and contributed to the interpretation of the results. R.N. calibrated the data. All authors have reviewed, discussed and commented on the manuscript.

Funding Open access funding provided by Universität zu Köln.

Competing interests The authors declare no competing interests.

Additional information

Supplementary information The online version contains supplementary material available at https://doi.org/10.1038/s41586-021-04294-5.

Correspondence and requests for materials should be addressed to Dominik $A$. Riechers. Peer review information Nature thanks Jeremy Darling, Eduaro González-Alfonso and the other, anonymous, reviewer for their contribution to the peer review of this work. Peer reviewer reports are available.

Reprints and permissions information is available at http://www.nature.com/reprints. 


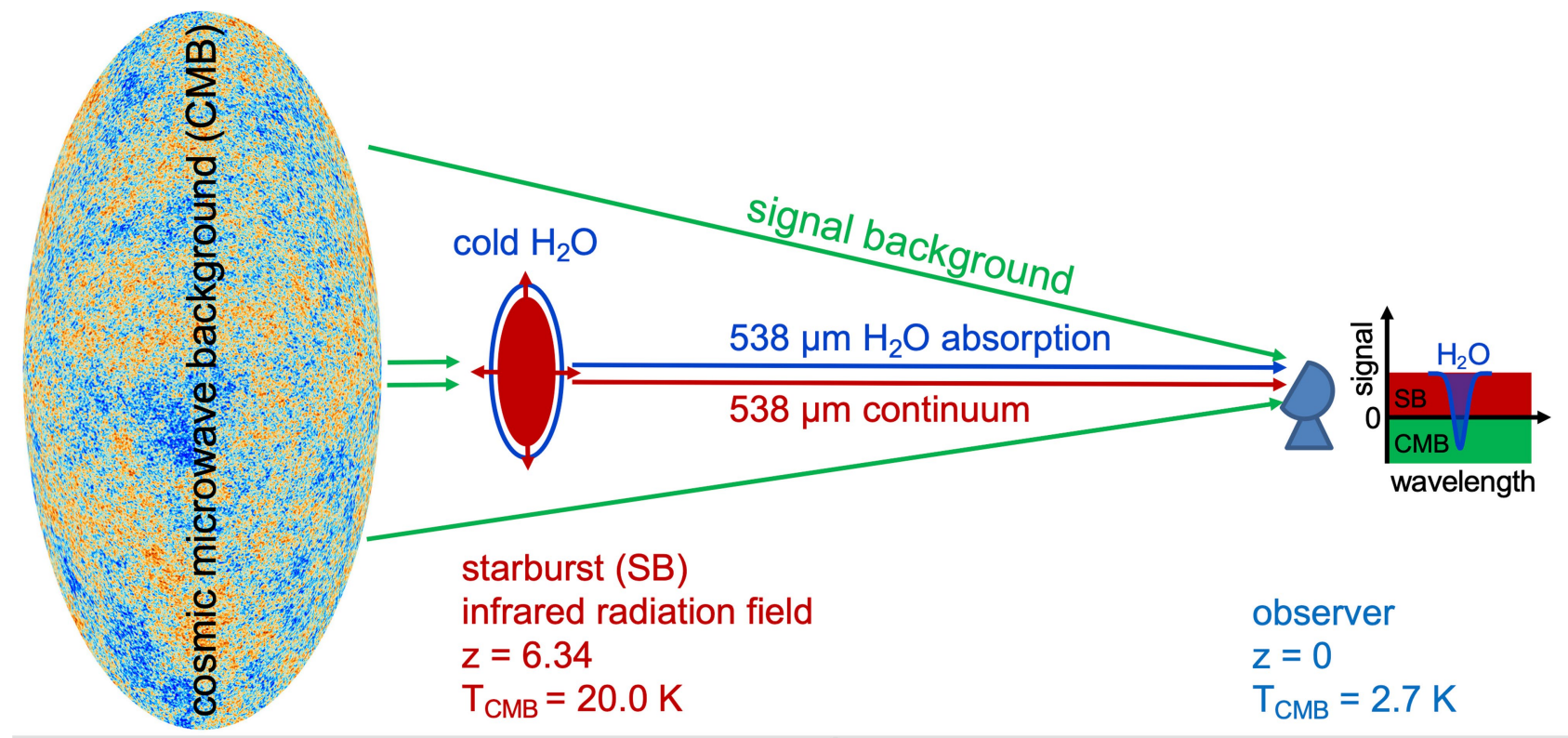

\section{Step 1 (no net $\mathrm{H}_{2} \mathrm{O}$ signal):}

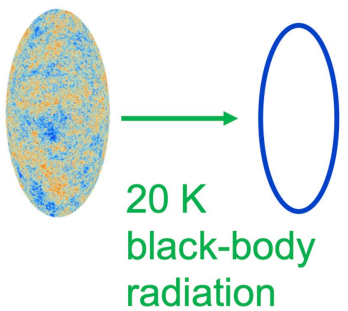

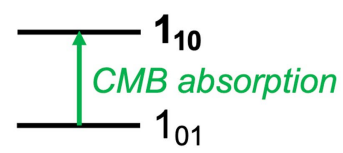

\section{$\mathrm{H}_{2} \mathrm{O}$ energy levels}

$\Delta \mathrm{E} / \mathrm{k}_{\mathrm{B}}=26.7 \mathrm{~K}$

thermal level

populations $\left(\mathrm{T}_{\mathrm{ex}}=\mathrm{T}_{\mathrm{CMB}}\right)$

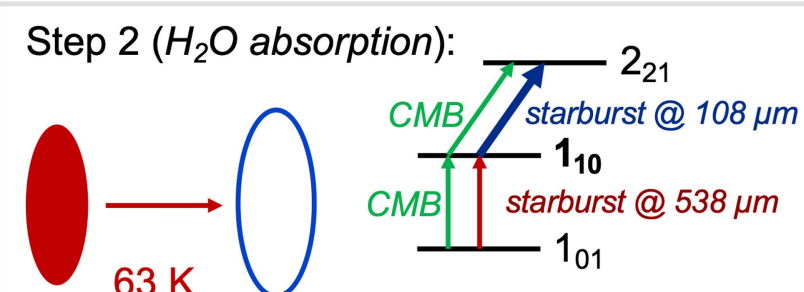

$63 \mathrm{~K}$ grey-body radiation

$\tau_{538 \mu \mathrm{m}}<\tau_{108 \mu \mathrm{m}}$
$\mathrm{H}_{2} \mathrm{O}$ energy levels $538 \mu \mathrm{m}$ : N photons $108 \mu \mathrm{m}: \mathrm{M}>\mathrm{N}$ photons $\rightarrow \mathrm{T}_{\mathrm{ex}}<\mathrm{T}_{\mathrm{CMB}}$
Extended Data Fig. 1 | Combined effect of CMB absorption and starburst radiation field on the strength of the $\mathrm{H}_{2} \mathrm{O}\left(\mathbf{1}_{10}-\mathbf{1}_{01}\right)$ line in HFLS3. Top, the cold $\mathrm{H}_{2} \mathrm{O}$ vapour is exposed to the $\mathrm{CMB}$ radiation field ${ }^{4}$, which has the shape of a black-body function $\left(T_{\mathrm{CMB}}=20.0 \mathrm{~K}\right.$ at $\left.z=6.34\right)$, and the starburst infrared radiation field, which has the shape of a grey-body function $\left(T_{\text {dust }}=63 \mathrm{~K}\right)$. NOEMA observed the signal in contrast to the $\mathrm{CMB}$ and therefore detects only the dust emission from the starburst and the $\mathrm{H}_{2} \mathrm{O}$ line, but not the $\mathrm{CMB}$ itself (which therefore fills the region below zero flux density, as seen by the telescope). Bottom left, as the energy-level difference for the $\mathrm{H}_{2} \mathrm{O}\left(1_{10}-1_{01}\right)$ line is only $26.7 \mathrm{~K}$, there are sufficient $\mathrm{CMB}$ photons at $z=6.34$ to thermalize the level population between both levels, such that $T_{\mathrm{ex}}$ is the same as $T_{\mathrm{CMB}}$ in equilibrium. Therefore $\mathrm{no}_{2} \mathrm{O}$ emission or absorption will be observed, despite the presence of a 'seed' population in the upper level. Bottom right, the radiation field of the starburst alters the level populations towards increased higher-level populations. Owing to the grey-body shape of its spectral energy distribution, more photons are available at $108 \mu \mathrm{m}$ to increase the $2_{21}$ level population from the $1_{10}$ state than there are $538-\mu \mathrm{m}$ photons available to increase the $1_{10}$ level population from the $1_{01}$ state, relative to the 'seed' population provided by the absorption of CMB photons. Therefore the relative population of the $1_{10}$ and $1_{01}$ levels is lower than in thermal equilibrium, such that the resulting $T_{\text {ex }}$ is lower than $T_{\text {CMB }}$. As a result, the $\mathrm{H}_{2} \mathrm{O}\left(1_{10}-1_{01}\right)$ line is observed in absorption towards the $\mathrm{CMB}$ due to the negative temperature contrast-as observed towards HFLS3. 


\section{Article}

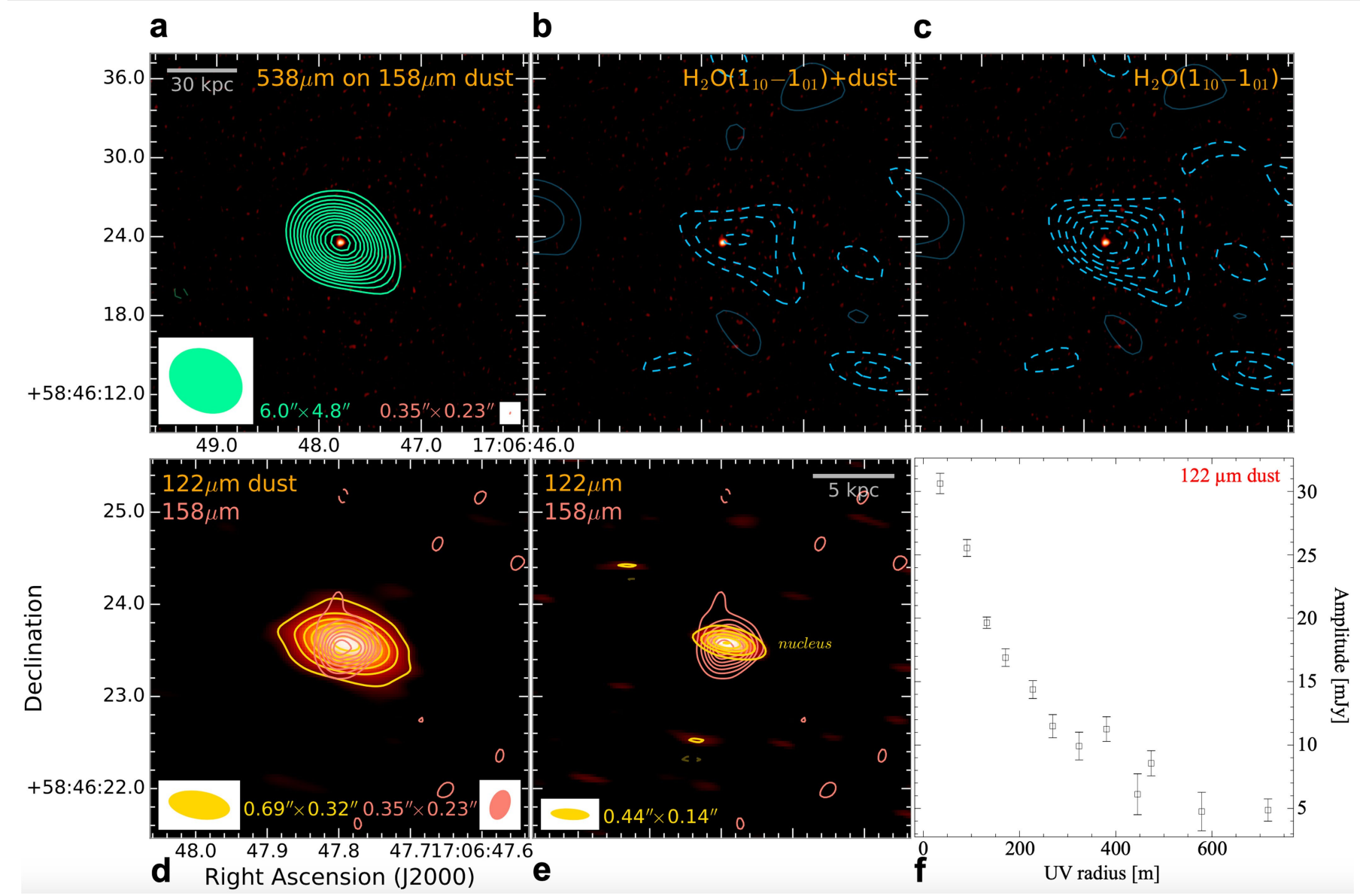

Extended Data Fig. $2 \mid \mathrm{H}_{2} \mathrm{O}$ line emission integrated moment $\mathrm{O}$ and continuum maps of HFLS3. a-c, $\mathrm{H}_{2} \mathrm{O}$ contour maps (blue) before (b) and after (c) continuum subtraction, and local continuum (a, green contours) at the wavelength of the $\mathrm{H}_{2} \mathrm{O}$ line, overlaid on the $158-\mu \mathrm{m}$ continuum (intensity scale $)^{5} \cdot \mathrm{H}_{2} \mathrm{O}$ emission is integrated over the central $395 \mathrm{~km} \mathrm{~s}^{-1}(100 \mathrm{MHz})$. d-e, Rest-frame 122- $\mu \mathrm{m}$ continuum emission (orange contours and intensity scale) as a proxy for the 108 - $\mu$ m continuum size, showing the full emission (d), and the compact nuclear region that accounts for two-thirds of the emission at higher resolution (e), overlaid with $158-\mu \mathrm{m}$ contours (red) for orientation.f, Radially averaged visibility amplitude as a function of interferometer baseline length for the data in $\mathbf{d}$ and $\mathbf{e}$. The radial profile of the visibility amplitude (binned to 50 -m steps, with $1 \sigma$ error bars) shows that the $122-\mu \mathrm{m}$ dust emission is clearly resolved. Observed-frame $538-\mu \mathrm{m}$ continuum contours (a) are shown in steps of $1 \sigma=22.5 \mu \mathrm{Jy} \mathrm{beam}^{-1}$, starting at $\pm 3 \sigma . \mathrm{H}_{2} \mathrm{O}$ contours $(\mathbf{b}, \mathbf{c})$ are shown in steps of $1 \sigma=0.0375 \mathrm{Jy} \mathrm{kms}^{-1}$ beam $^{-1}$, starting at $\pm 2 \sigma$. Contours of $122 \mu \mathrm{m}(\mathbf{d}, \mathbf{e})$ are shown in steps of $+/-10 \sigma$ and $+/-5 \sigma$, where $1 \sigma=229$ and $374 \mu \mathrm{Jy} \mathrm{beam}^{-1}$, respectively. Contours of $158 \mu \mathrm{m}(\mathbf{d}, \mathbf{e})$ are shown in steps of $3 \sigma$, starting at $\pm 5 \sigma$, where $1 \sigma=400 \mu \mathrm{Jy}_{\text {beam }}{ }^{-1}$ (all uncertainties are r.m.s.). Negative intensity contours are dashed. 
Extended Data Table 1| Summary of $T_{\mathrm{CMB}}(z)$ measurements

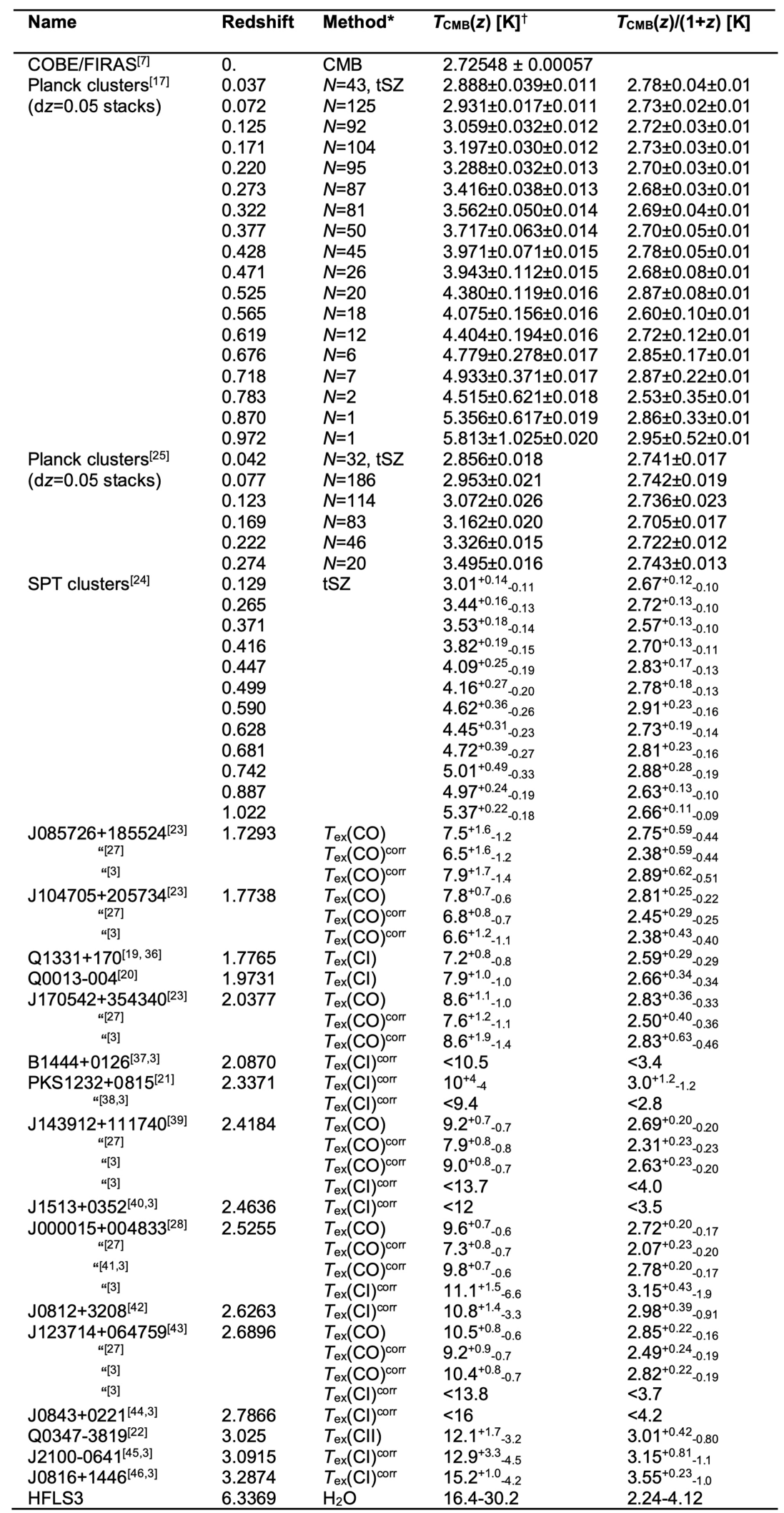

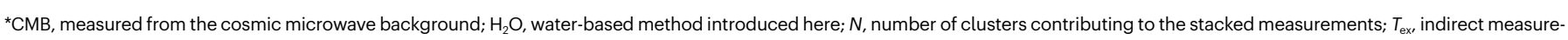
ment based on the excitation temperature of $\mathrm{CO},[\mathrm{Cl}]$ or [CII] of ultraviolet absorption lines along the lines of sight to background quasars, with model-based excitation corrections applied where indicated (different values indicate model corrections from different authors to the same data); $\mathrm{tSZ}$, measured from the thermal Sunyaev-Zel'dovich effect in galaxy clusters.

'Where provided, the second set of error bars indicates systematic uncertainties. Other error bars correspond to statistical uncertainties. 